University of Nebraska - Lincoln

DigitalCommons@University of Nebraska - Lincoln

Faculty Publications from the Department of Engineering Mechanics

$11-2004$

\title{
Edge-Cracked Orthotropic Bimaterial Butt Joint under Antiplane Singularity
}

Xiangfa Wu

Department of Engineering Mechanics, University of Nebraska-Lincoln, xfwu@unlserve.unl.edu

Yuris A. Dzenis

Department of Engineering Mechanics, University of Nebraska-Lincoln, ydzenis@unl.edu

Emrah Gokdag

University of Houston

Follow this and additional works at: https://digitalcommons.unl.edu/engineeringmechanicsfacpub

Part of the Mechanical Engineering Commons

Wu, Xiangfa; Dzenis, Yuris A.; and Gokdag, Emrah, "Edge-Cracked Orthotropic Bimaterial Butt Joint under Antiplane Singularity" (2004). Faculty Publications from the Department of Engineering Mechanics. 26. https://digitalcommons.unl.edu/engineeringmechanicsfacpub/26

This Article is brought to you for free and open access by the Mechanical \& Materials Engineering, Department of at DigitalCommons@University of Nebraska - Lincoln. It has been accepted for inclusion in Faculty Publications from the Department of Engineering Mechanics by an authorized administrator of DigitalCommons@University of Nebraska - Lincoln. 


\title{
Edge-Cracked Orthotropic Bimaterial Butt Joint under Antiplane Singularity
}

\author{
Xiang-Fa Wu, Yuris A. Dzenis \\ Department of Engineering Mechanics, Center for Materials Research and Analysis, \\ University of Nebraska-Lincoln, Lincoln, NE 68588-0526, USA \\ Email:xfwu@unlserve.unl.edu (X.-F.Wu)
}

\section{Emrah Gokdag}

Department of Mechanical Engineering, University of Houston, Houston, TX 77204-4792, USA

\begin{abstract}
Explicit elastic solutions are given for an edge-cracked orthotropic bimaterial butt joint under antiplane singularity of a screw dislocation and a line-force. During the procedure, conformal mapping and known dislocation solution are utilized for constructing the fundamental solution to the present problem. Stress intensity factor (SIF) and energy release rate (ERR) of the edge-cracked butt joint are given in closed-form. In limiting cases, results provided in this work cover those in literature.
\end{abstract}

Keywords: Interfacial edge-crack; butt joint; screw dislocation; stress intensity factor (SIF); conformal mapping; orthotropic bimaterials

\section{Introduction}

With increasing applications of ceramics, composite laminates, and joints in aerospace and aeronautical engineering, interfacial cracks between bonded anisotropic media have been studied extensively in past decades. A number of researchers contributed significantly in this interesting area such as the works by Willis (1971), Ting (1986), Qu and Bassani (1989), Suo (1990), Wu (1990), Gao et al. (1992) and Hwu (1993), etc., in which the given solutions are limited to cracks between two dissimilar anisotropic half-planes. However, interfacial cracks often initiate and grow near free edges of composite laminates and joints where high stress concentration exists due to the mismatch of Poisson's ratios of the component materials, and the bonding strength is relatively low. These interfacial edge-cracks usually bear length comparable to structural dimensions, e.g. layer thickness. So far, except for very limited cases such as those bimaterial strips with semi-infinite interfacial cracks (Wu et al., 2002, 2003), inplane interfacial edge-cracks are only solved by means of numerical methods, e.g. FEM, $\mathrm{BEM}$, or methods of singular integral equations, etc. Nevertheless, in the case of antiplane deformation, a quite few closed-from solutions have been achieved in literature. For example, by means of dual integral equations, Choi et al. (1994) dealt with an interfacial edge-crack between two orthotropic quarter-planes. Lee and Earmme (2000) reinvestigated the same problem using conformal mapping technique.

In reality, bonded bimaterial strips such as composite butt joints are used extensively in composite engineering due to their unique structures and cost-efficiency. Under external loadings, interfacial edge-cracking is the main failure mode of these structures. Recently, Wu and Dzenis (2002a, 2002b) and Wu et al. (2003) have considered edge-cracks in isotropic/orthotropic strips and joints. Closedform solutions of these cracks have been presented, which could be used for the study of scaling effect on the corresponding fracture 
parameters. In this study we further consider the closed-form elastic solution to a double edgecracked bimaterial butt joint under antiplane singularity of a screw dislocation and a lineforce. Its exact solution may serve as Green's function for constructing solutions to strips with multiple cracks under arbitrary antiplane loadings. The closed-form solution for this study is obtained by determining the dislocation solution to the bimaterial butt joint through conformal mapping, similar to those by Shiue et al. (1989), Lee and Earmme (2000), and Wu et al. (2002). Limiting geometrical cases are further explored to show the scaling effect on the fracture parameters.

\section{Formulation and solution procedure}

Let us first determine the complex potential for an orthotropic bimaterial strip under antiplane singularity. The materials discussed in this study are assumed as orthotropic with the $\xi \eta$-plane as a mirror plane. In this case the inplane and antiplane deformations are decoupled, and then they can be treated independently. Under antiplane deformation, the displacement and stress of an anisotropic body may be expressed in terms of an analytic function $\varphi(\zeta)($ Suo, 1990), i.e.

$$
\begin{aligned}
& u_{3}(\xi, \eta)=-2 \operatorname{Im}[B \varphi(\zeta)], \\
& \sigma_{23}(\xi, \eta)=-2 \operatorname{Re}\left[\varphi^{\prime}(\zeta)\right], \\
& \sigma_{13}(\xi, \eta)=2 \operatorname{Re}\left[\mu \varphi^{\prime}(\zeta)\right],
\end{aligned}
$$

where the prime denotes the derivative with respect to the complex variable $\zeta=\xi+\mu \eta, B$ and $\mu$ are two material constants defined as

$$
\begin{aligned}
& B=\sqrt{S_{44} S_{55}-S_{45}^{2}}, \\
& \mu=\left[S_{45}+i \sqrt{S_{44} S_{55}-S_{45}^{2}}\right] / S_{55},
\end{aligned}
$$

$S_{\mathrm{ij}}$ are the shear compliances of the materials. In the case of orthotropic materials, $B=\sqrt{S_{44} S_{55}}$, and $\mu=i \sqrt{S_{44} / S_{55}}$ is a positive imaginary.
Consider an antiplane singularity located in an orthotropic bimaterial, as shown in Fig. 1. Without loss of generality, the singularity is assumed located in the lower half-plane at $\zeta_{0}=\xi_{0}+\mu_{2} \eta_{0} \quad\left(\eta_{0}<0\right)$, and $\xi$ and $\eta$ being the coordinates of the material point. The complex displacement potential for this problem has been given in literature (Suo, 1990), i.e.

$\varphi(\zeta)=\left\{\begin{array}{c}2 B_{2} /\left(B_{1}+B_{2}\right) \varphi_{0}(\zeta),(\eta>0) \\ \varphi_{0}(\zeta)-\left(B_{1}-B_{2}\right) /\left(B_{1}+B_{2}\right) \bar{\varphi}_{0}(\zeta), \\ (\eta<0)\end{array}\right.$

where

$\varphi_{0}(\zeta)=q \ln \left(\zeta-\zeta_{0}\right)$

Here subscripts 1 and 2 signify the quantities relating materials 1 and 2 respectively, the overbar ( $)$ denotes the complex conjugate, and the quantity $q$ is defined as

$q=-b /\left(4 \pi B_{2}\right)+i p /(4 \pi)$,

where $b$ is the Burgers vector of the screw dislocation and $p$ is the line-force.

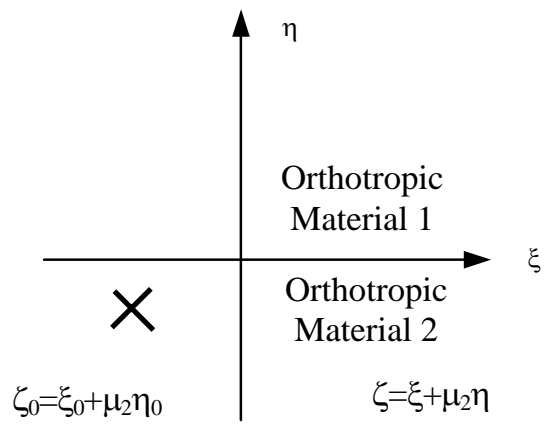

Fig. 1 Orthotropic bimaterial ( $\zeta$-plane) under antiplane singularity

\subsection{Solution to screw dislocation in orthotropic bimaterial butt joint}

Now let us consider a screw dislocation located in a bimaterial strip made of two boned dissimilar orthotropic strips of width $W$, as shown in Fig. 2(a). The dislocation is assumed 
located in the lower strip at $z_{0}=x_{0}+\mu_{2} y_{0}\left(y_{0}<0\right), x$ and $y$ being the coordinates of the material point, and the joint surfaces being traction-free. This problem can be solved as a periodic problem, as $\left.x_{0}+2 W\right)+\mu_{2} y_{0}$ in the bimaterial strip of width $2 W$, respectively.

Since the displacement potential is analytic in the strips above and below, the solution to the

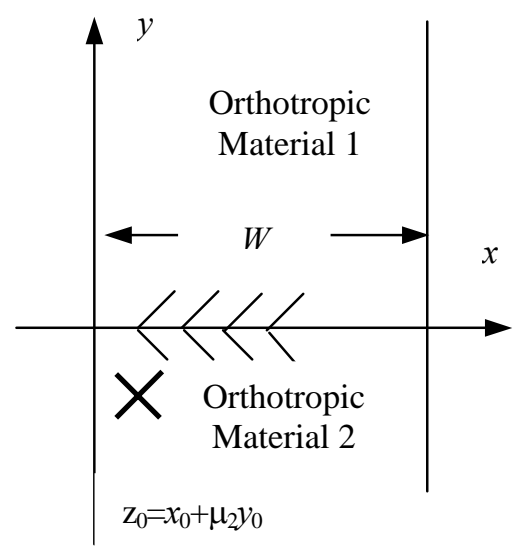

(a)

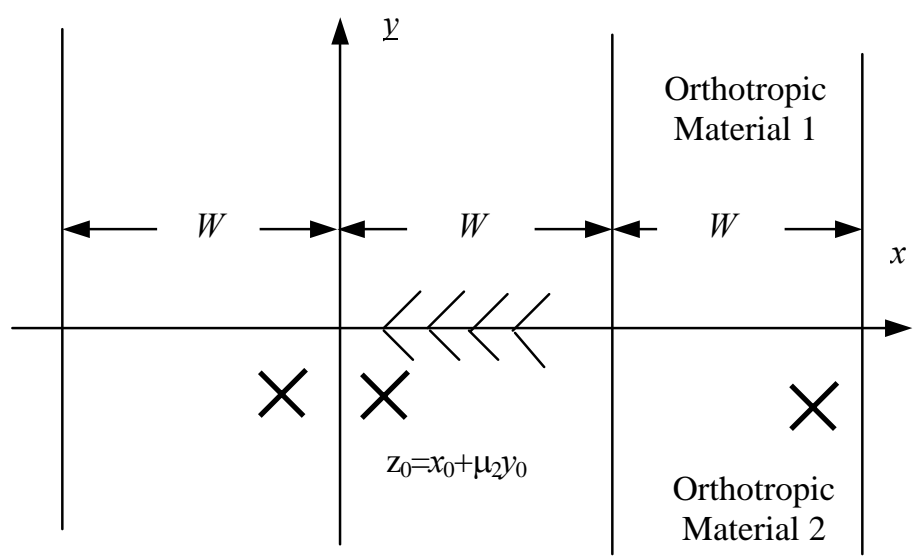

(b)

Fig. 2 Orthotropic bimaterial under antiplane singularities

(a) Butt joint (z-plane), (b) bimaterial medium (z-plane)

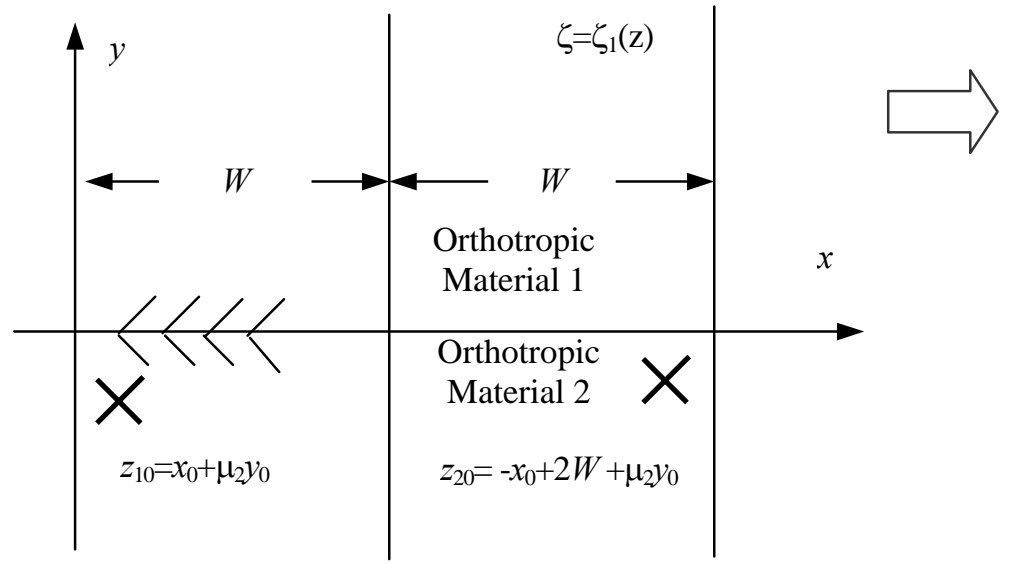

(a)

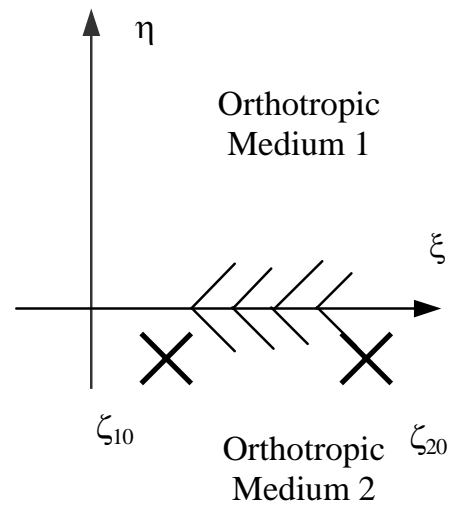

(b)

Fig. 3 Orthotropic bimaterial under two antiplane singularities

(a) Butt joint ( $z$-plane); (b) bimaterial ( $\zeta$-plane)

shown in Fig. 2(b), with dislocations of quantity $q$ periodically located at $\mathrm{z}_{\mathrm{n}}=\left(x_{0}+2 N W\right)+\mu_{2} y_{0}$ and $\mathrm{Z}_{\text {-n }}=\left(-x_{0}+2 N W\right)+\mu_{2} y_{0}(N=0, \pm 1, \pm 2 \ldots \pm \infty)$. The representative elemental strip (RES) is shown in Fig. 3(a), where two equivalent dislocations of quantity $q$ are located symmetrically at $\mathrm{z}_{1}=x_{0}+\mu_{2} y_{0}$ and $\mathrm{z}_{2}=(-$
RES may be constructed by means of conformal mapping technique as follows. Introduce the conformal mapping

$$
\zeta=\tan [\pi(z-W) /(2 W)],\left(z=x+\mu_{2} y\right)
$$


which maps the RES, as shown in Fig.3(a), onto two bond half-planes with two cuts $(\infty,-\mathrm{i})$ and (i, $\infty)$ at the imaginary axis, and locations of two screw dislocations $\mathrm{z}_{1}$ and $\mathrm{z}_{2}$ are mapped respectively onto

$$
\zeta_{10}=-\cot \left[\pi z_{0} /(2 W)\right], \zeta_{10}=\cot \left[\pi \bar{z}_{0} /(2 W)\right] .
$$

Due to the periodic property, the physical quantities at two sides of the cuts $(\infty,-i)$ and (i, $\infty)$ bear the same values, thus we can directly utilize the dislocation solutions (3) and (4) to construct the strip solution such that

$$
f(z)=\left\{\begin{array}{cc}
2 B_{2} /\left(B_{1}+B_{2}\right) f_{0}(z), & (y>0) \\
f_{0}(z)-\left(B_{1}-B_{2}\right) /\left(B_{1}\right. & \left.+B_{2}\right) \bar{f}_{0}(z), \\
& (y<0)
\end{array}\right.
$$

where

$$
\begin{aligned}
f_{0}(z) & =q\left\{\ln \left\{\cot \left[\pi z_{0} /(2 W)\right]-\cot [\pi z /(2 W)]\right\}\right. \\
& \left.+\ln \left\{-\cot \left[\pi \bar{z}_{0} /(2 W)\right]-\cot [\pi z /(2 W)]\right\}\right\},
\end{aligned}
$$

and $q$ is defined by (5).

With the aid of (1), solutions (8) and (9) determine the entire displacement and stress fields in the bimaterial butt joint under the antiplane singularity at $z_{0}=x_{0}+\mu_{2} y_{0}$.

\subsection{Solution to edge-cracked bimaterial butt joint under antiplane singularity}

Now let us consider a screw dislocation in an edge-cracked butt joint made of two dissimilar orthotropic strips as shown in Fig. 4(a), where $W, a, b$, and $\mathrm{z}_{0}\left(z_{0}=x_{0}+\mu_{2} y_{0}, y_{0}<0\right)$ denote the strip width, locations of crack tips, and the location of the antiplane singularity, respectively. As discussed in Section 2.1, we try to construct the solution by using the known solutions (8) and (9) and conformal mapping technique. Here we introduce the new conformal mapping

$$
\begin{aligned}
& \zeta=\frac{2 W}{\pi} \tan ^{-1} \sqrt{\frac{\sin ^{2}[\pi z /(2 W)]-\sin ^{2}[\pi a /(2 W)]}{\sin ^{2}[\pi b /(2 W)]-\sin ^{2}[\pi z /(2 W)]}}, \\
& \left(z=x+\mu_{2} y\right)
\end{aligned}
$$

which maps the edge-cracked butt joint, as shown in Fig. 4(a), onto a strip, as shown in Fig. 4(b). The screw dislocation located at $z_{0}=$ $x_{0}+\mu_{2} y_{0}$ in the $\mathrm{z}$-plane is mapped onto

$$
\zeta_{0}=\frac{2 W}{\pi} \tan ^{-1} \sqrt{\frac{\sin ^{2}\left[\pi z_{0} /(2 W)\right]-\sin ^{2}[\pi a /(2 W)]}{\sin ^{2}[\pi b /(2 W)]-\sin ^{2}\left[\pi z_{0} /(2 W)\right]}},
$$

in the mapped strip ( $\zeta$-plane).

With the help of solution (9), we have

$$
\begin{aligned}
f_{0}(z)= & q\left\{\ln \left[X\left(z_{0}\right)-X(z)\right]\right. \\
& \left.+\ln \left[-\overline{X\left(z_{0}\right)}-X(z)\right]\right\},
\end{aligned}
$$

where

$$
X(z)=\sqrt{\frac{\sin ^{2}[\pi b /(2 W)]-\sin ^{2}[\pi z /(2 W)]}{\sin ^{2}[\pi z /(2 W)]-\sin ^{2}[\pi a /(2 W)]}} .
$$

Substitution of (12) and (13) into (8) yields the displacement potential of the cracked butt joint.

Within the framework of linear fracture mechanics, the most important parameters are the stress intensity factor (SIF) and the energy release rate (ERR). Here we only consider the mode-III fracture parameters $\left(K_{\mathrm{III}}\right.$ and $\left.G_{\mathrm{III}}\right)$, which are evaluated as

$$
\begin{aligned}
& K_{I I I}=\lim _{x \rightarrow x_{0}}-\sqrt{2 \pi\left(x-x_{0}\right)} \operatorname{Re}\left[f^{\prime}(x)\right], \\
& G_{I I I}=\left(B_{1}+B_{2}\right) K_{I I I}^{2} / 4,
\end{aligned}
$$

where $x_{0}$ denotes the crack tip at the $x$-axis, and $\operatorname{Re}($ ) stands for the real part of an analytic function. In the present case, substitution of (8), (12), and (13) into (14) yields the SIFs at crack tips $a$ and $b$ respectively as 


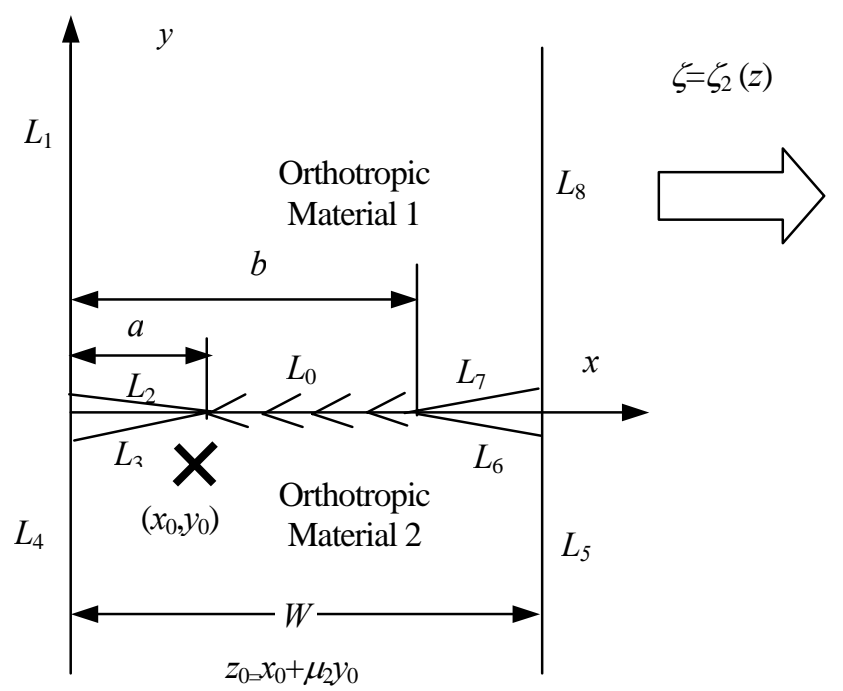

(a)

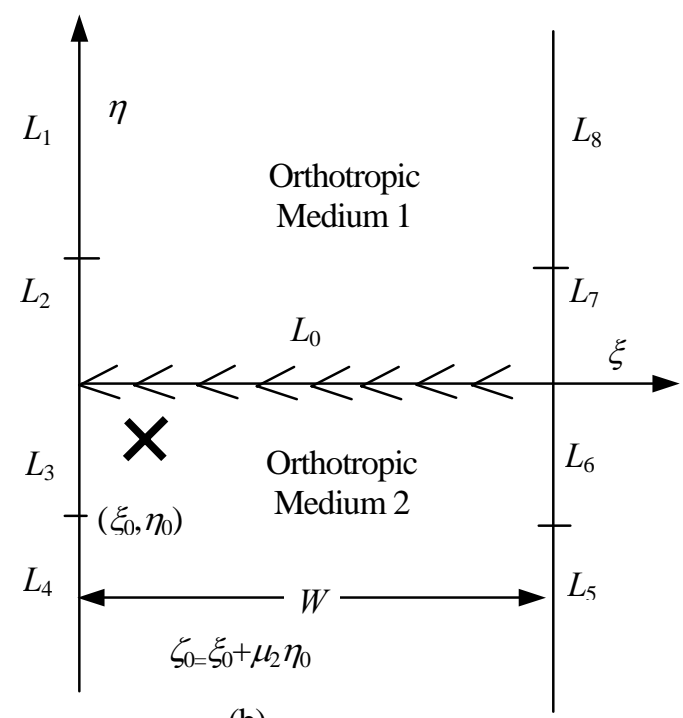

(b)

Fig. 4 Orthotropic bimaterial butt joint under antiplane singularity (a) Edge-cracked butt joint ( $z$-plane), (b) butt joint ( $\zeta$-plane)

$$
\begin{aligned}
K_{I I I}^{a} & =\lim _{x \rightarrow a} \sqrt{2 \pi(x-a)}\left[f^{\prime}(x)+\overline{f^{\prime}(x)}\right] \\
& =4 \pi B_{2} /\left[\left(B_{1}+B_{2}\right) \sqrt{\pi a}\right] \\
& \times \sqrt{\frac{\pi a / W \sin (\pi a / W)}{\sin ^{2}[\pi b /(2 W)]-\sin ^{2}[\pi a /(2 W)]}} \\
& \times \operatorname{Re}\left(-q \sqrt{\frac{\sin ^{2}[\pi b /(2 W)]-\sin ^{2}\left[\pi z_{0} /(2 W)\right]}{\sin ^{2}\left[\pi z_{0} /(2 W)\right]-\sin ^{2}[\pi a /(2 W)]}}\right),
\end{aligned}
$$

and

$$
\begin{aligned}
& K_{I I I}^{b}=\lim _{x \rightarrow b} \sqrt{2 \pi(b-x)}\left[f^{\prime}(x)+\overline{f^{\prime}(x)}\right] \\
& \quad=4 \pi B_{2} /\left[\left(B_{1}+B_{2}\right) \sqrt{\pi b}\right] \\
& \quad \times \sqrt{\frac{\pi b / W \sin (\pi b / W)}{\sin ^{2}[\pi b /(2 W)]-\sin ^{2}[\pi a /(2 W)]}} \\
& \quad \times \operatorname{Re}\left(-q \sqrt{\frac{\sin ^{2}\left[\pi z_{0} /(2 W)\right]-\sin ^{2}[\pi a /(2 W)]}{\sin ^{2}[\pi b /(2 W)]-\sin ^{2}\left[\pi z_{0} /(2 W)\right]}}\right) .
\end{aligned}
$$

The corresponding ERRs $\left(G_{\text {III }}\right)$ may be evaluated by substituting (15) and (16) into the second formula of (14). Furthermore, relations
(15) and (16) can serve as Green's functions for constructing the SIFs $\left(K_{\text {III }}\right)$ and ERRs $\left(G_{\text {III }}\right)$ of edge-cracked orthotropic butt joints under arbitrary antiplane loadings.

\section{Examples: joint surface under action of isolated line-forces}

Here we consider a special case of the butt joint as shown in Fig. 5 where two isolated lineforces are located at the crack surface and the

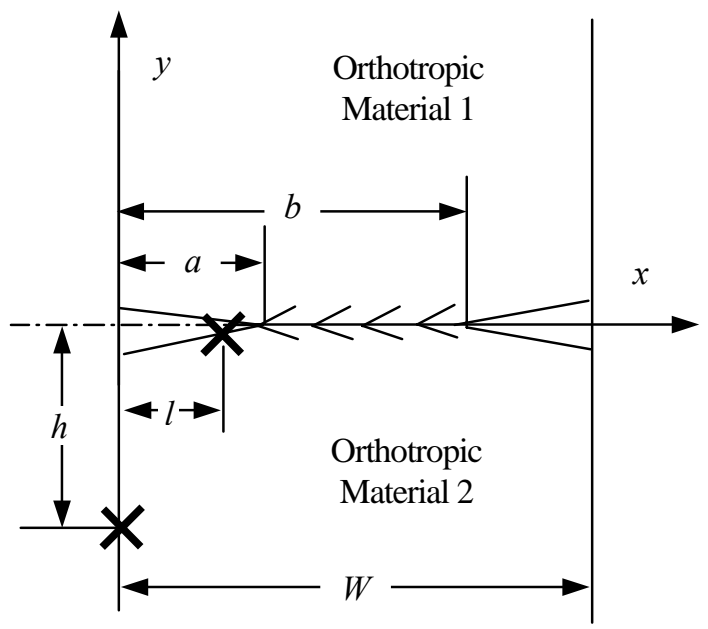

Fig. 5 Orthotropic bimaterial butt joint under action of two line-forces 
free-edge surface of the lower strip, respectively. With the help of definition (5), we assume that the singularities of quantity $q_{1}=\mathrm{i} P / 4 \pi$ located at $z_{1}=l-0 i$ and $q_{2}=\mathrm{i} Q / 4 \pi$ located at $z_{2}=-h i$, respectively. Substituting quantities $q_{1}$ and $q_{2}$, and the corresponding dislocation coordinates $z_{1}$ and $\mathrm{z}_{2}$ into (15) and (16), we obtain the SIFs $\left(K_{\text {III }}\right)$ as

$$
\begin{aligned}
K_{I I I}^{a} & =B_{2} /\left[\left(B_{1}+B_{2}\right) \sqrt{\pi a}\right] \\
& \times \sqrt{\frac{\pi a / W \sin (\pi a / W)}{\sin ^{2}[\pi b /(2 W)]-\sin ^{2}[\pi a /(2 W)]}} \\
& \times\left\{P \sqrt{\frac{\sin ^{2}[\pi b /(2 W)]-\sin ^{2}[\pi / /(2 W)]}{\sin ^{2}[\pi a /(2 W)]-\sin ^{2}[\pi l /(2 W)]}}\right. \\
& \left.+Q \sqrt{\frac{\sin ^{2}[\pi b /(2 W)]+\sinh ^{2}[\pi h /(2 W)]}{\sin ^{2}[\pi a /(2 W)]+\sinh ^{2}[\pi h /(2 W)]}}\right\},
\end{aligned}
$$

and

$$
\begin{aligned}
K_{I I I}^{b} & =B_{2} /\left[\left(B_{1}+B_{2}\right) \sqrt{\pi b}\right] \\
& \times \sqrt{\frac{\pi b / W \sin (\pi b / W)}{\sin ^{2}[\pi b /(2 W)]-\sin ^{2}[\pi a /(2 W)]}} \\
& \times\left\{P \sqrt{\frac{\sin ^{2}[\pi a /(2 W)]-\sin ^{2}[\pi l /(2 W)]}{\sin ^{2}[\pi b /(2 W)]-\sin ^{2}[\pi l /(2 W)]}}\right. \\
& \left.+Q \sqrt{\frac{\sin ^{2}[\pi a /(2 W)]+\sinh ^{2}[\pi h /(2 W)]}{\sin ^{2}[\pi b /(2 W)]+\sinh ^{2}[\pi h /(2 W)]}}\right\} .
\end{aligned}
$$

By letting $P=0$, and $h \rightarrow \infty$, we have

$$
\begin{aligned}
K_{I I I}^{a} & =B_{2} /\left(B_{1}+B_{2}\right)(Q / W) \\
& \times \sqrt{\frac{W \sin (\pi a / W)}{\sin ^{2}[\pi b /(2 W)]-\sin ^{2}[\pi a /(2 W)]}}, \\
K_{I I I}^{b} & =B_{2} /\left(B_{1}+B_{2}\right)(Q / W) \\
& \times \sqrt{\frac{W \sin (\pi b / W)}{\sin ^{2}[\pi b /(2 W)]-\sin ^{2}[\pi a /(2 W)]}},
\end{aligned}
$$

which are the SIFs $\left(K_{\text {III }}\right)$ of the edge-cracked joint with constant antiplane traction $Q / W$ acting at the infinity of the lower strip.

Now let us consider the limiting cases of solution (17). By letting $b \rightarrow W$, as shown in Fig. $6(a)$, we obtain the SIF ( $\left.K_{\text {III }}\right)$ of an interfacial edge-crack in a bimaterial strip, i.e.

$$
\begin{aligned}
& K_{I I I}=2 B_{2} /\left[\left(B_{1}+B_{2}\right) \sqrt{\pi a}\right] \sec [\pi a /(2 W)] \\
& \times\left\{P \sqrt{\frac{\pi a /(2 W) \tan [\pi a /(2 W)]}{\tan ^{2}[\pi a /(2 W)]-\tan ^{2}[\pi l /(2 W)]}}\right. \\
& \left.+Q \sqrt{\frac{\pi a /(2 W) \tan [\pi a /(2 W)]}{\tan ^{2}[\pi a /(2 W)]+\tanh ^{2}[\pi h /(2 W)]}}\right\},
\end{aligned}
$$

which accords to the solution given by Wu et al. (2003).

Furthermore, by letting $W \rightarrow \infty$ in (20) as shown in Fig. $6(b)$, we derive the SIF $\left(K_{\text {III }}\right)$ of an interfacial edge-crack between two bonded dissimilar orthotropic quarter-planes, i.e.

$$
\begin{aligned}
K_{I I I} & =2 B_{2} /\left[\left(B_{1}+B_{2}\right) \sqrt{\pi a}\right] \\
& \times\left(a P / \sqrt{a^{2}-l^{2}}+a Q / \sqrt{a^{2}+h^{2}}\right),
\end{aligned}
$$

which agrees with those given by Choi et al. (1994) and Lee and Earmme (2000). In general, the mapping function $\varsigma=z^{2}$ used by Lee and Earmme (2000) does not map two bonded anisotropic quarter-planes $\left(z=x+\mu y, \mu=\lambda_{1}+\mathrm{i} \lambda_{2}\right.$, $\lambda_{1} \neq 0$ and $\lambda_{2}>0$ ) onto two bonded half-planes with a semi-infinite cut along the negative real axis, thus the solution provided by Lee and Earmme (2000) only holds in the case of orthotropic materials.

As shown in Fig. 6(c), if only considering an isolated line-force $P$ located at the lower crack surface, letting $a \rightarrow \infty$ and simultaneously keeping $c=(W-a)$ and $l_{0}=(a-l)$ constant in (20), we then obtain the SIF $\left(K_{\text {III }}\right)$ of a semi-infinite interfacial crack heading towards a free surface as

$$
K_{I I I}=\frac{2 P}{\sqrt{\pi l_{0}}} \frac{B_{2}}{B_{1}+B_{2}} \frac{l_{0}+c}{\sqrt{c\left(l_{0}+2 c\right)}},
$$


where $l_{0}$ is the distance from the crack tip to the line-force location.

If further letting $a \rightarrow \infty$ in (21) or $c \rightarrow \infty$ in (22), we obtain the $\operatorname{SIF}\left(K_{\mathrm{III}}\right)$ for a semi-infinite interfacial crack between two bonded dissimilar orthotropic half-planes as evaluated by inserting (17)-(23) into the second formula in (14).

Consequently, by letting $B_{1}=B_{2}$, all relations derived above cover those for strips made of homogenous orthotropic (or isotropic) materials.

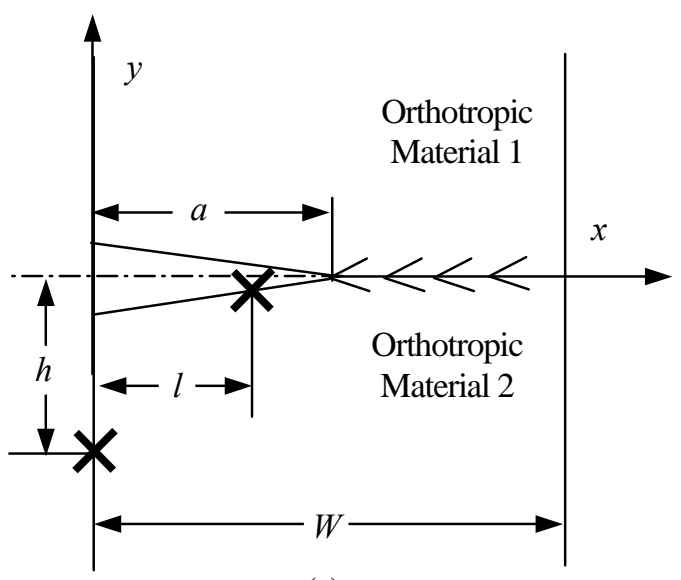

(a)

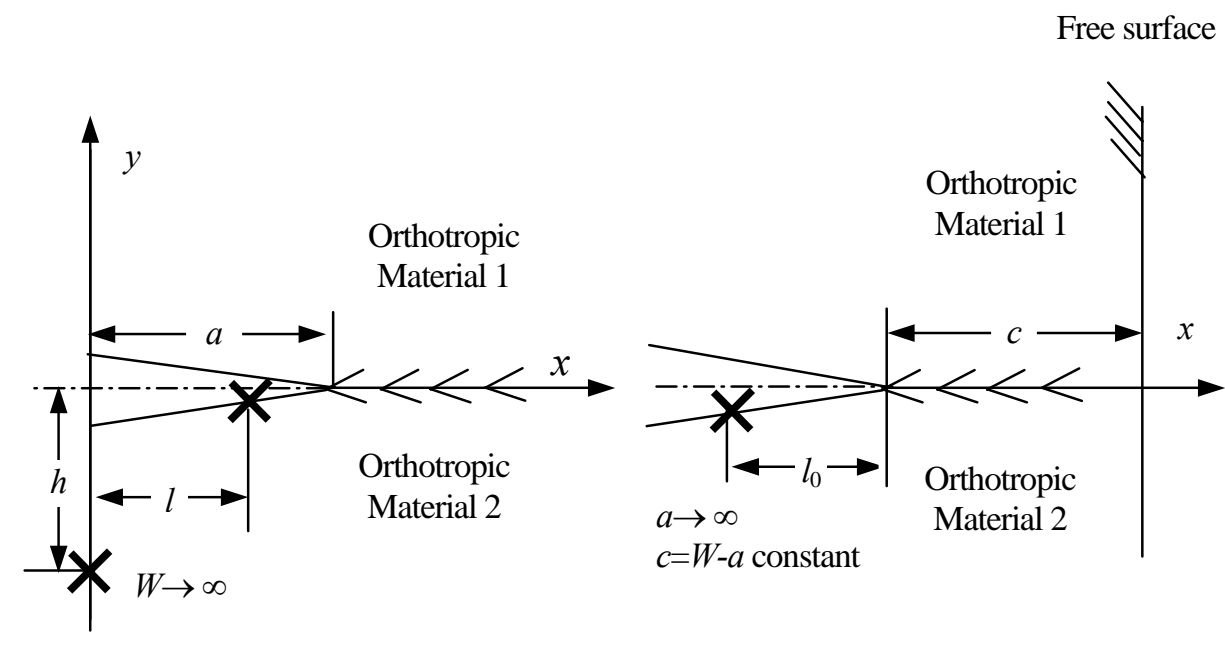

(b)

(c)

Fig. 6 Cracked bimaterial under action of line-forces

(a) Edge-cracked strip, (b) edge-cracked half-planes, (c) crack heading towards a free surface.

$$
K_{I I I}=\sqrt{\frac{2}{\pi l_{0}}} \frac{B_{2}}{B_{1}+B_{2}} P,
$$

which is corresponding to the relation given by Suo (1990). The ERR $\left(G_{\text {III }}\right)$ of each crack configuration aforementioned can be easily

\section{Conclusions}

Under antiplane deformation, displacement potentials of orthotropic materials satisfy the modified Laplace equations, whose solutions can be expressed in terms of analytic functions. In the case of edge-cracked bimaterial butt joint 
under antiplane singularity, by properly selecting conformal mapping function, we obtain the closed-form displacement potential, $\operatorname{SIF}\left(K_{\text {III }}\right)$, and ERR $\left(G_{\text {III }}\right)$ etc., which are applicable for scaling analysis of the fracture parameters. In limiting geometrical cases, the current results exactly cover those in literature. All relations derived in this study may be used as Green's functions for the determination of the SIFs $\left(K_{\text {III }}\right)$ and ERRs $\left(G_{\text {III }}\right)$ of edge-cracked butt joints with arbitrary antiplane forces acting out of the crack surfaces, which are generally difficult to be solved directly using method of dual integral equations.

\section{Acknowledgement}

Partial support of this work by the U. S. Air Force Office of Scientific Research and the U.S. Army Research Office is gratefully acknowledged.

\section{References}

[1] Choi, S. R., Chong, C.H., Chai, Y.S. Interfacial edge crack in two bonded dissimilar orthotropic quarter planes under antiplane shear, International Journal of Fracture, 67 (1994) 143-150

[2] Gao, H., Abbudi, M., Barnett, D. M. On interfacial crack-tip field in anisotropic elastic solids, Journal of the Mechanics and Physics of Solids, 40 (1992) 393-416

[3] Hwu, C.B. Explicit solutions for collinear interface crack problems, International Journal of Solids and Structures, 30 (1993) 301-312

[4] Lee, K. W., Earmme, Y. Y. An interfacial edge crack in anisotropic bimaterial under antiplane singularity, International Journal of Fracture, 104 (2000) 15-22

[5] Qu, J., Bassani, J. L. Finite crack on bimaterial and bicrystal interface, Journal of the Mechanics and Physics of Solids, 37 (1989) 435-453

[6] Shiue, S. T., Hu, C. T., Lee, S. Elastic interaction between screw dislocation and a welded surface crack in composite materials, Engineering Fracture Mechanics, 33 (1989) 697-706

[7] Suo, Z. Singularities, interfaces and cracks in dissimilar anisotropic media. Proceedings of the Royal of Society of London, A427 (1990) 331-358

[8] Ting, T. C. T. Explicit solution and invariance of the singularities at an interface crack in anisotropic composites, International Journal of Solids and Structures, 22 (1986) 965-983

[9] Willis, J. R. Fracture mechanics of interfacial cracks, Journal of the Mechanics and Physics of Solids, 19 (1971) 353-368

[10] Wu, K. C. Stress intensity factor and energy release rate for interfacial cracks between dissimilar anisotropic materials, ASME Journal of Applied Mechanics, 57 (1990) 882-886

[11] Wu, X. F., Dzenis, Y. A. Closed-form solution for a mode-III interfacial crack between two bonded dissimilar elastic strips, Mechanics Research Communications, 29 (2002a) 407-412

[12] Wu, X. F., Dzenis, Y. A. Closed-form solution for the size of plastic zone in an edge-cracked strip, International Journal of Engineering Science, 40 (2002b) 1751-1759

[13] Wu, X. F, Lilla, E., Zou, W. S. A semi-infinite interfacial crack between two bonded dissimilar elastic strips, Archive of Applied Mechanics, 72 (2002), 630-636

[14] Wu, X. F., Dzenis, Y. A., Fan, T. Y. Two semi-infinite interfacial cracks between two bonded dissimilar elastic strips, International Journal of Engineering Science, 41 (2003) 1699-1710

[15] Wu, X. F., Dzenis, Y. A., Zou, W. S. Interfacial edge crack between two bonded dissimilar orthotropic strips under anti-plane point loading, Zeitschrift fur Angewandte Mathematik und Mechanik (ZAMM), 83 (2003) 419-422 